\title{
Why rogue waves occur atop abrupt depth transitions
}

\author{
Yan $\operatorname{Li}^{1,2} \dagger$, Samuel Draycott ${ }^{3}$, Yaokun Zheng ${ }^{4}$, Zhiliang $\operatorname{Lin}^{4}$, Thomas \\ A.A. Adcock ${ }^{1}$, Ton S. van den Bremer ${ }^{1,5}$ \\ ${ }^{1}$ Department of Engineering Science, University of Oxford, Parks Road, Oxford OX1 3PJ, UK \\ ${ }^{2}$ Department of Energy and Process Engineering, Norwegian University of Science and \\ Technology, N-7491 Trondheim, Norway \\ ${ }^{3}$ Department of Mechanical, Aerospace and Civil Engineering, University of Manchester, \\ Manchester M13 9PL, UK \\ ${ }^{4}$ State Key Laboratory of Ocean Engineering, School of Naval Architecture, Ocean and Civil \\ Engineering, Shanghai Jiao Tong University, Shanghai 200240, China \\ ${ }^{5}$ Faculty of Civil Engineering and Geosciences, Delft University of Technology, 2628 CD, Delft, \\ The Netherlands
}

(Received $\mathrm{xx}$; revised $\mathrm{xx}$; accepted $\mathrm{xx}$ )

Abrupt depth transitions (ADTs) have recently been identified as potential causes of 'rogue' ocean waves. When stationary and (close-to) normally distributed waves travel into shallower water over an ADT, distinct spatially localized peaks in the probability of extreme waves occur. These peaks have been predicted numerically, observed experimentally, but not explained theoretically. Providing this theoretical explanation using a leading-order-physics-based statistical model, we show, by comparing to new experiments and numerical simulations, the peaks arise from the interaction between linear free and second-order bound waves, also present in the absence of the ADT, and new second-order free waves generated due to the ADT.

\section{Introduction}

Different physical mechanisms have been proposed to explain so-called 'rogue' waves on the ocean surface (Kharif et al. 2008; Dysthe et al. 2008; Onorato et al. 2013; Adcock \& Taylor 2014; Dudley et al. 2019). Rogue waves are typically defined as large waves whose height exceeds the significant wave height by a factor 2-2.2 (Kharif et al. 2008). Such waves are of increased interest if they occur more frequently than predicted by a Gaussian process. A useful proxy for rogue waves therefore is the excess kurtosis of the free surface relative to a Gaussian process (Mori \& Janssen 2006). Physical mechanisms proposed include random linear dispersive focusing enhanced by weak bound-wave nonlinearity (Fedele et al. 2016), modulational or Benjamin-Feir instability in deep water(Benjamin \& Feir 1967; Janssen 2003), and abrupt depth transitions (ADTs) among others.

Recently, several authors have shown that rogue waves can form at the top of ADTs for waves in shallow to intermediate depth, as reviewed in Trulsen et al. (2020). In the experiments of Trulsen et al. (2012), local peaks of skewness and kurtosis occurred a short distance after a 1:20 underwater slope when waves travelled from a deeper to a shallower domain (see also Zhang et al. (2019)). Similar peaks have been observed experimentally for shoals (Trulsen et al. 2020; Ma et al. 2014) and steps (Bolles et al. 2019). Local peaks in skewness and kurtosis have also been predicted using reduced-form nonlinear evolution

$\dagger$ Email address for correspondence: yan.li@ntnu.no 
equations, such as the KdV equation for variable shallow depth (Sergeeva et al. 2011; Majda et al. 2019) and the Boussinesq equations (Gramstad et al. 2013; Zhang et al. 2019; Kashima et al. 2014), and by fully nonlinear numerical simulations of the water wave equations (Ducrozet \& Gouin 2017; Viotti \& Dias 2014; Zheng et al. 2020; Zhang \& Benoit 2021). The magnitude of the peaks is greatest when the ADT is infinitely steep, i.e. a step (Zheng et al. 2020). Peaks in skewness and kurtosis only occur for sufficiently shallow depths (Trulsen et al. 2020; Zheng et al. 2020), corresponding to a depth beyond the applicability of the nonlinear Schrödinger equation (Zeng \& Trulsen 2012), which correctly predicts the absence of peaks in deeper water (Lawrence et al. 2021).

Two hypotheses have been proposed to explain these peaks. According to the first, ADTs place the system out of equilibrium; the peaks are the response of a system that rapidly adjusts to a new equilibrium driven by nonlinear (third and higher-order in steepness) processes (Trulsen 2018; Viotti \& Dias 2014). According to the second hypothesis, the peaks are formed by second-order effects in steepness (Gramstad et al. 2013; Ducrozet \& Gouin 2017; Zhang et al. 2019; Zheng et al. 2020), but the mechanism by which is not clear.

We develop a statistical model based on the second-order theory for wave propagation over a step developed in $\mathrm{Li}$ et al. (2021b) and validated experimentally in $\mathrm{Li}$ et al. (2021a) that can accurately predict the magnitude and location of the peaks in kurtosis atop ADTs. Our model confirms the validity of the second hypothesis and demonstrates the underlying mechanism is one of the interplay of linear free and second-order bound waves, which are also present in the absence of the ADT, and the second-order free waves generated due to the ADT.

\section{Theoretical model}

\subsection{Deterministic model ( $\mathrm{Li}$ et al. 2021b)}

Our starting point is the deterministic model for wave group propagation over a step of ( $\mathrm{Li}$ et al. 2021b). We consider weakly nonlinear unidirectional water waves on the surface of a constant density fluid, ignoring surface tension and viscosity, so that the fluid satisfies potential flow. The ADT (cf. figure 1) takes the form of a discontinuity or 'step' in water depth $h(x)$ at $x=0$, where $h$ changes from $h_{d}$ for $x<0$ to a shallower value $h_{s}$ for $x>0$ with $x$ denoting the horizontal axis. We consider intermediate water depths $(\mathcal{O}(k h)=1$ with $k$ the wavenumber) and leading-order approximations to the water wave equations. Specifically, as in the classical statistical models of Tayfun (1980, 1986), the solutions are valid up to second order in steepness $\epsilon=k_{0} A_{0}$, where $k_{0}$ and $A_{0}$ denote the characteristic wavenumber and amplitude, respectively, and narrow-banded or valid up to first order in the dimensionless bandwidth parameter, defined as $\delta=1 /\left(k_{0} \sigma_{0}\right)$ with $\sigma_{0}$ the characteristic group length.

\subsubsection{Incident wave field}

The surface elevation of the incident wave field travelling towards the ADT can be obtained from a combined Stokes and multiple-scales expansion (e.g., Mei et al. (1989)):

$$
\begin{aligned}
\zeta(x, t) & =\zeta^{(1)}(x, t)+\zeta^{(2)}(x, t) \text { for } x<0, \text { with } \\
\zeta^{(1)}(x, t) & =A \cos \psi_{0}(x, t) \text { and } \zeta^{(2)}(x, t)=k_{0} A^{2}\left[C_{20, b}+C_{22, b} \cos 2 \psi_{0}(x, t)\right],
\end{aligned}
$$

where the superscripts denote the order in $\epsilon, A(x, t)$ the envelope, $\psi_{0}=k_{0} x-\omega_{0} t+\theta_{0}$ the phase, with $k_{0}$ the carrier wavenumber and $\omega_{0}=\omega\left(k_{0}, h_{d}\right)$ the angular velocity obeying linear dispersion, $\omega(k, h)=\sqrt{g k \tanh k h}$ with $g$ gravity, and $\theta_{0}$ the phase. The 


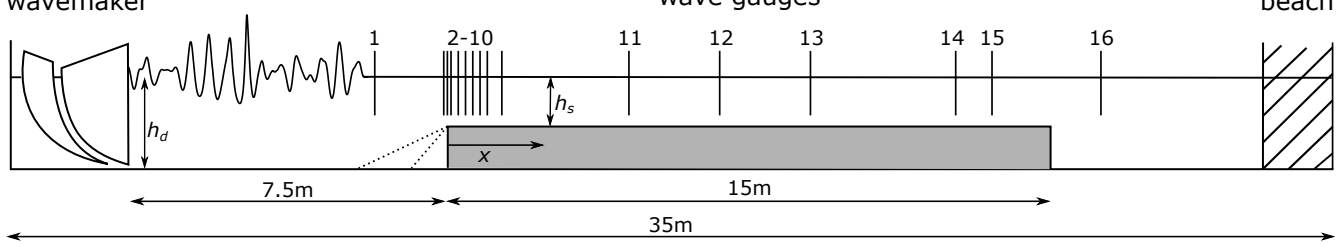

FiguRE 1. Experimental set-up.

envelope $A(x, t)=A\left(x / c_{g 0}-t\right)$ travels at the group velocity $c_{g 0}=c_{g}\left(k_{0}, h_{d}\right)$, with $c_{g}(k, h)=(\omega /(2 k))(1+2 k h / \sinh 2 k h)$. The coefficients of the bound second-order subharmonic and super-harmonic waves are (e.g., Mei et al. (1989)):

$$
\begin{aligned}
& C_{20, b}(k h)=\left[\left(2 g h-c_{g}^{2}\right) /(2 \sinh 2 k h)+2 g c_{g} / \omega\right] /\left[4\left(c_{g}^{2}-g h\right)\right], \\
& C_{22, b}(k h)=\cosh k h\left(2 \cosh ^{2} k h+1\right) /\left(4 \sinh ^{3} k h\right),
\end{aligned}
$$

where $C_{20, b}\left(k_{0} h_{d}\right)$ and $C_{22, b}\left(k_{0} h_{d}\right)$ in $(2.1)$ and we have further assumed the envelope is long relative to the water depth (as in Mei et al. (1989)).

\subsubsection{Transmitted wave field}

Upon reaching the ADT $(x=0)$, the incident wave field is reflected and transmitted. We focus on the latter here. At first order in $\epsilon$, the carrier wave on the shallower side travels at speed $c_{g 0 s}=c_{g}\left(k_{0 s}, h_{s}\right)$, where $k_{0 s}$ denotes the wavenumber that can be found by solving $\omega_{0}=\omega\left(k_{0 s}, h_{s}\right)$ and corresponds to a shorter wavelength. In addition, evanescent waves are generated in the vicinity of the step that vanish exponentially with distance away from the step. At second order in $\epsilon$, the sub-harmonic and superharmonic bound waves associated with the transmitted wave field must change magnitude at the step, and additional free sub-harmonic and super-harmonic waves are generated, which, respectively, travel at the shallow-water speed $\sqrt{g h_{s}}$ or satisfy the linear dispersion relation for $\omega\left(k_{20 s}, h_{s}\right)=2 \omega_{0}$ with $k_{20 s}$ the wavenumber of the super-harmonic waves.

Based on Massel (1983), who derived expressions for both linear and second-order super-harmonic components for a monochromatic wave, and $\mathrm{Li}$ et al. (2021b), who extended this work to narrow-banded wavepackets, the surface elevation on the shallower side (i.e. for $x>0$ ) is given by (Li et al. 2021b):

$$
\begin{aligned}
\zeta_{s}(x, t) & =\zeta_{s}^{(1)}(x, t)+\zeta_{s}^{(2)}(x, t) \text { for } x>0, \text { with } \\
\zeta_{s}^{(1)}(x, t) & =A_{s}(x, t) \cos \psi_{0 s}+\zeta_{E s}^{(1)}, A_{s}(x, t)=\left|T_{0}\right| A\left(x / c_{g 0 s}-t\right), \\
\zeta_{s}^{(2)}(x, t) & =\zeta_{s}^{(20)}+\zeta_{s}^{(22)}, \\
\zeta_{s}^{(20)}(x, t) & =C_{20, b} k_{0 s} A_{s}^{2}+C_{20}, k_{0 s}\left|T_{0}\right|^{2} A^{2}\left(x / \sqrt{g h_{s}}-t\right)+\zeta_{E s}^{(20)}, \text { and } \\
\zeta_{s}^{(22)}(x, t) & =C_{22, b} k_{0 s} A_{s}^{2} \cos \left(2 \psi_{0 s}\right)+C_{22}, f k_{0 s}\left|T_{0}\right|^{2} A^{2}\left(x / c_{g 22 s}-t\right) \cos \left(2 \psi_{0 s}+\psi_{22, f}\right)+\zeta_{E s}^{(22)},
\end{aligned}
$$

in which $T_{0}$ denotes the complex transmission coefficient, the subscripts $E, b$ and $f$ the evanescent, bound and free waves, respectively. In the first-order term, $\psi_{0 s}(x, t)=$ $\psi_{0}(x, t)+\left(k_{0 s}-k_{0}\right) x+\theta_{T_{0}}$ with $\theta_{T_{0}}$ denoting the phase shift due the step $\left(\theta_{T_{0}}=\arg \left(T_{0}\right)\right)$. The second-order bound wave coefficients can be evaluated from (2.2): $C_{20, b}\left(k_{0 s} h_{s}\right)$, $C_{22, b}\left(k_{0 s} h_{s}\right)$. The second-order free wave coefficients are given by $C_{20, f}=\left|T_{20, f}\right| /\left|T_{0}\right|^{2}$ and $C_{22, f}=2 \omega_{0}^{2}\left|T_{22, f}\right| /\left(g k_{0 s}\left|T_{0}\right|^{2}\right)$ with $T_{20}$, and $T_{22, f}$ the (complex) coefficients for the sub- and super-harmonic free wave, respectively. $T_{0}, T_{20}$, and $T_{22, f}$ are obtained from the boundary conditions at the step (see Li et al. (2021b) for details). In the second-order terms, $c_{g 22 s}=c_{g}\left(k_{20 s}, h_{s}\right)$ is the group velocity of the free super-harmonic envelope, 
$\psi_{22, f}(x)=k_{20 s} x-2 k_{0 s} x+\theta_{T_{22, f}}-2 \theta_{T_{0}}$ denotes the phase of the free super-harmonic waves relative to the bound super-harmonic waves with $\theta_{T_{22, f}}$ the phase shift of the free super-harmonic waves upon transmission $\left(\theta_{T_{22, f}}=\arg \left(T_{22, f}\right)\right)$. To obtain tractable solutions, Li et al. (2021b) ignore forcing by (small) products linear evanescent waves.

\subsection{A new statistical model}

To develop a statistical model based on the deterministic model of Li et al. $(2021 b)$ outlined in $\S 2.1$, we assume a normally distributed linear incident field $\tilde{\zeta}^{(1)}$ with variance $\mu_{0}$, which is both stationary and homogeneous (for $x<0$ ). Specifically, we define a Rayleigh-distributed envelope $\tilde{A}$ and a uniformly distributed phase $\tilde{\psi}$, so that $\tilde{\zeta}^{(1)}=$ $\tilde{A} \cos (\tilde{\psi})$. Tildes denote random variables. The incident wave field on the deeper side $(x<0)$ becomes (cf. $(2.1))$ :

$$
\tilde{\zeta}=\tilde{\zeta}^{(1)}+\tilde{\zeta}^{(2)} \text { for } x<0, \tilde{\zeta}^{(1)}=\tilde{A} \cos \tilde{\psi} \text {, and } \tilde{\zeta}^{(2)}=k_{0} \tilde{A}^{2}\left[C_{20, b}+C_{22, b} \cos 2 \tilde{\psi}\right] . \quad[2.4 \mathrm{a}, \mathrm{b}, \mathrm{c}]
$$

Neglecting the effect of evanescent waves, we obtain on the shallower side (cf. (2.3)):

$$
\begin{aligned}
& \tilde{\zeta}_{s}=\tilde{\zeta}_{s}^{(1)}+\tilde{\zeta}_{s}^{(2)} \text { for } x>0, \text { with } \\
& \tilde{\zeta}_{s}^{(1)}=\left|T_{0}\right| \tilde{A} \cos \left(\tilde{\psi}_{0 s}\right) \text { and } \tilde{\zeta}_{s}^{(2)}(x)=\tilde{\zeta}_{s}^{(20)}(x)+\tilde{\zeta}_{s}^{(22)}(x), \\
& \tilde{\zeta}_{s}^{(20)}(x)=k_{0 s}\left|T_{0}\right|^{2} \tilde{A}^{2}\left(C_{20, b}+C_{20, f} R_{20}(x)\right), \tilde{\psi}_{0 s}=\tilde{\psi}+\left(k_{0 s}-k_{0}\right) x+\theta_{T_{0}} \text {, and } \\
& \tilde{\zeta}_{s}^{(22)}(x)=k_{0 s}\left|T_{0}\right|^{2} \tilde{A}^{2}\left[C_{22, b} \cos \left(2 \tilde{\psi}_{0 s}\right)+C_{22, f} R_{22}(x) \cos \left(2 \tilde{\psi}_{0 s}+\psi_{22, f}(x)\right)\right],
\end{aligned}
$$

where $R_{20}(x)$ and $R_{22}(x)$ are envelope functions, obtained by expanding the envelope of the sub- and super-harmonic waves about the center of the transmitted envelope, respectively. They can be expressed in terms of the focused deterministic envelope $A$ as:

$$
R_{20}(x)=A^{2}\left(x / \sqrt{g h_{s}}-x / c_{g 0 s}\right) / A^{2}(0), R_{22}(x)=A^{2}\left(x / c_{g 22 s}-x / c_{g 0 s}\right) / A^{2}(0), \quad[2.6 \mathrm{a}, \mathrm{b}]
$$

where $A(0)$ is the central magnitude of the deterministic envelope. The focused deterministic envelope can be obtained from the energy spectrum $S(\omega)$ by $A(t)=$ $\int \sqrt{2 S(\omega)} \cos \left(\left(\omega-\omega_{0}\right) t\right) \mathrm{d} \omega$. For a Gaussian deterministic envelope $A=a_{0} \exp \left(-c_{g 0}^{2}\left(x / c_{g 0 s}-\right.\right.$ $\left.t)^{2} /\left(2 \sigma_{0}^{2}\right)\right)$, we obtain $R_{20}(x)=\exp \left[-\left(c_{g 0} x / \sqrt{g h_{s}}-c_{g 0} x / c_{g 0 s}\right)^{2} / \sigma_{0}^{2}\right]$ and $R_{20}(x)=$ $\exp \left[-\left(c_{g 0} x / c_{g 22 s}-c_{g 0} x / c_{g 0 s}\right)^{2} / \sigma_{0}^{2}\right]$. A long distance away from the step, $R_{20}, R_{22} \rightarrow 0$, and we recover the standard homogeneous result for constant depth (e.g., Tayfun (1986)).

\subsubsection{Statistical properties}

The skewness $s=\left\langle\left(\tilde{\zeta}_{s}-m\right)^{3}\right\rangle / v^{3 / 2}$ and kurtosis $\kappa=\left\langle\left(\tilde{\zeta}_{s}-m\right)^{4}\right\rangle / v^{2}$ of the random surface elevation can be directly obtained from $(2.5)$ with $m=\left\langle\tilde{\zeta}_{s}\right\rangle, v=\left\langle\left(\tilde{\zeta}_{s}-m\right)^{2}\right\rangle$ and $\langle\cdots\rangle$ the combined expectation operator of random variables $\tilde{A}$ and $\tilde{\psi}$,

$$
\begin{aligned}
s & =6 k_{0 s} \sqrt{\mu_{0 s}} C_{2}(x)+\mathcal{O}\left(\mu_{0 s}^{3 / 2}\right), \\
\kappa & =3+24 k_{0 s}^{2} \mu_{0 s}\left(\kappa_{2, b}+\kappa_{2, b f}(x)+\kappa_{2, f}(x)\right)+\mathcal{O}\left(\mu_{0 s}^{2}\right),
\end{aligned}
$$

where $\mu_{0 s}=\left|T_{0}\right|^{2} \mu_{0}$ denotes the variance of the surface elevation on the shallower side, $C_{2}(x)=C_{20, b}+C_{22, b}+C_{20, f} R_{20}(x)+C_{22, f} R_{22}(x) \cos \psi_{22, f}(x), \kappa_{2, b}$ captures the contribution to kurtosis by bound waves, $\kappa_{2, f}$ by free waves and $\kappa_{2, b f}$ by their combination:

$$
\begin{aligned}
\kappa_{2, b} & =3 C_{20, b}^{2}+4 C_{20, b} C_{22, b}+3 C_{22, b}^{2}, \\
\kappa_{2, f}(x) & =3 C_{20, f}^{2} R_{20}^{2}+4 C_{20, f} C_{22, f} R_{20} R_{22} \cos \psi_{22, f}+3 C_{22, f}^{2} R_{22}^{2}, \\
\kappa_{2, b f}(x) & =\left(6 C_{20, b}+4 C_{22, b}\right)\left(C_{20, f} R_{20}+C_{22, f} R_{22} \cos \psi_{22, f}\right) .
\end{aligned}
$$


From $(2.5)$, we can also obtain a second-order accurate expression for wave crests $\tilde{\zeta}_{c}$ :

$$
\tilde{\zeta}_{c}(x)=\left|T_{0}\right| \tilde{A}+k_{0 s}\left|T_{0}\right|^{2} \tilde{A}^{2} C_{2}(x) .
$$

In non-dimensional form, $\tilde{\xi}_{c}=\tilde{\zeta}_{c} / H_{s s}$ with $H_{s s}=4 \sqrt{\mu_{0 s}}$ the significant wave height on the shallower side, the crest elevation has the probability density function (cf. Tayfun $(1980))$,

$$
f_{\tilde{\xi}_{c}}\left(\xi_{c}\right)=\frac{16 u \exp \left(-8 u^{2}\right)}{1+4 \epsilon_{s} C_{2}(x) u} \text { with } u=\frac{\sqrt{1+8 \epsilon_{s} C_{2}(x) \xi_{c}}-1}{4 \epsilon_{s} C_{2}(x)},
$$

and $\epsilon_{s}=k_{0 s} H_{s s} / 2$ measures steepness in a random sea. Eq. (2.11) can be integrated to obtain the exceedance probability (cf. Forristall (2000)),

$$
P\left(\tilde{\xi}_{c}>\xi_{c}\right)=\exp \left(-8 u^{2}\left(\xi_{c}\right)\right) \text {. }
$$

As $\epsilon_{s} \rightarrow 0$, we recover from (2.11) and (2.12) the Rayleigh distribution. If the contributions by the second-order free waves are neglected, which is valid in the absence of, or a long distance away from, a step, then $R_{20} \rightarrow 0$ and $R_{22} \rightarrow 0$, and (2.5), (2.7), $(2.8)$ and (2.10) reduce to the second-order accurate result for constant depth (Tayfun $1980,1986)$.

\subsubsection{Rogue wave generating mechanism}

As described in $§ 2.1 .2$, second-order sub- and super-harmonic free waves are released upon transmission over the ADT. On the shallower side, these free waves co-exist with the transmitted linear free and their second-order bound waves, which would also be present for constant depth. Compared to the second-order statistical model for constant depth (Tayfun 1980, 1986), the additional second-order free waves lead to an increased likelihood of large waves atop the ADT. The likelihood of large waves is enhanced by the presence of sub-harmonic free waves $\left(C_{20, f}>0\right)$ and beating between the bound and free super-harmonics, which results in local maxima ( $\mathrm{Li}$ et al. 2021b). Both these effects only occur near the top of an ADT, where the second-order free waves are initially generated and where their envelopes still overlap with the linear envelope. Since both sub-harmonics and super-harmonics propagate at different group speed from the linear envelope, they separate over a sufficient distance away from the step. This mechanism locally amplifies skewness (2.7), kurtosis (2.8), and exceedance probability of crests (2.12).

\section{Results}

\subsection{Experiments and numerical simulations}

To validate our statistical model, we have performed laboratory experiments and fully nonlinear numerical simulations. Experiments were carried out in the Coastal, Ocean and Sediment Transport (COAST) Laboratory at the University of Plymouth, UK. A schematic of the experimental set-up (and the numerical wave tank) is shown in Fig. 1. Two water depths on the deeper side were used: $h_{d}=0.55 \mathrm{~m}$ and $h_{d}=0.75 \mathrm{~m}$. Hence, the water depth on the shallower side $h_{s}=h_{d}-0.35 \mathrm{~m}$. The numerical wave tank (Zheng et al. 2020) is equivalent to the laboratory except that the computational domain has a total length of $50 \lambda_{0}$, where $\lambda_{0}$ denotes the carrier wavelength on the deeper side, with a length of $20 \lambda_{0}$ and $30 \lambda_{0}$ on the deeper $(x<0)$ and shallower sides $(x>0)$, respectively.

In both the experiments and numerics, the (linear) wavemaker was programmed to generate irregular waves based on JONSWAP spectra for different peak frequencies $f_{p}$ and water depths (peak enhancement factor $\gamma=3.3$ ). Four cases are examined with 
TABLE 1. Parameters of the laboratory experiments and fully nonlinear numerical simulations. The shallower water depth $h_{s}=h_{d}-0.35 \mathrm{~m}$, the bandwidth $\delta=1 /\left(k_{0} \sigma_{0}\right), H_{m 0}$ is the measured significant wave height of the entire signal (the table shows its value obtained at the first gauge on the deeper side), $H_{s}=4 \sqrt{\mu_{0}}$ is the significant wave height of the linearised surface elevation on the deeper side, and the steepness $\epsilon=k_{0} H_{s} / 2, \Gamma(k h)=\epsilon C_{22 b}(k h) /(k h)^{3}$ denotes a parameter that measures the degree of nonlinearity relative to depth (Toffoli et al. 2007), with $C_{22, b}$ defined by $(2.2 b)$, and $N_{1}$ and $N_{2}$ denote the total number of the random realisations per case in experiments and numerical simulations, respectively.

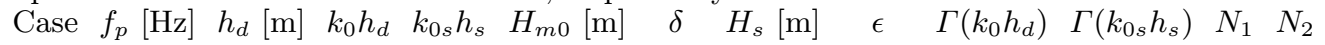

\begin{tabular}{ccccccccccccc}
\hline $\mathrm{A}$ & 0.80 & 0.55 & 1.6 & 0.79 & 0.046 & 0.12 & 0.040 & 0.057 & 0.0097 & 0.19 & 14 & 14 \\
$\mathrm{~B}$ & 0.60 & 0.55 & 1.0 & 0.57 & 0.035 & 0.24 & 0.029 & 0.027 & 0.019 & 0.26 & 14 & 14 \\
$\mathrm{C}$ & 0.70 & 0.75 & 1.6 & 1.0 & 0.078 & 0.22 & 0.069 & 0.074 & 0.013 & 0.12 & 8 & 14 \\
$\mathrm{D}$ & 0.55 & 0.75 & 1.1 & 0.76 & 0.079 & 0.22 & 0.069 & 0.052 & 0.036 & 0.20 & 9 & 14 \\
\hline
\end{tabular}

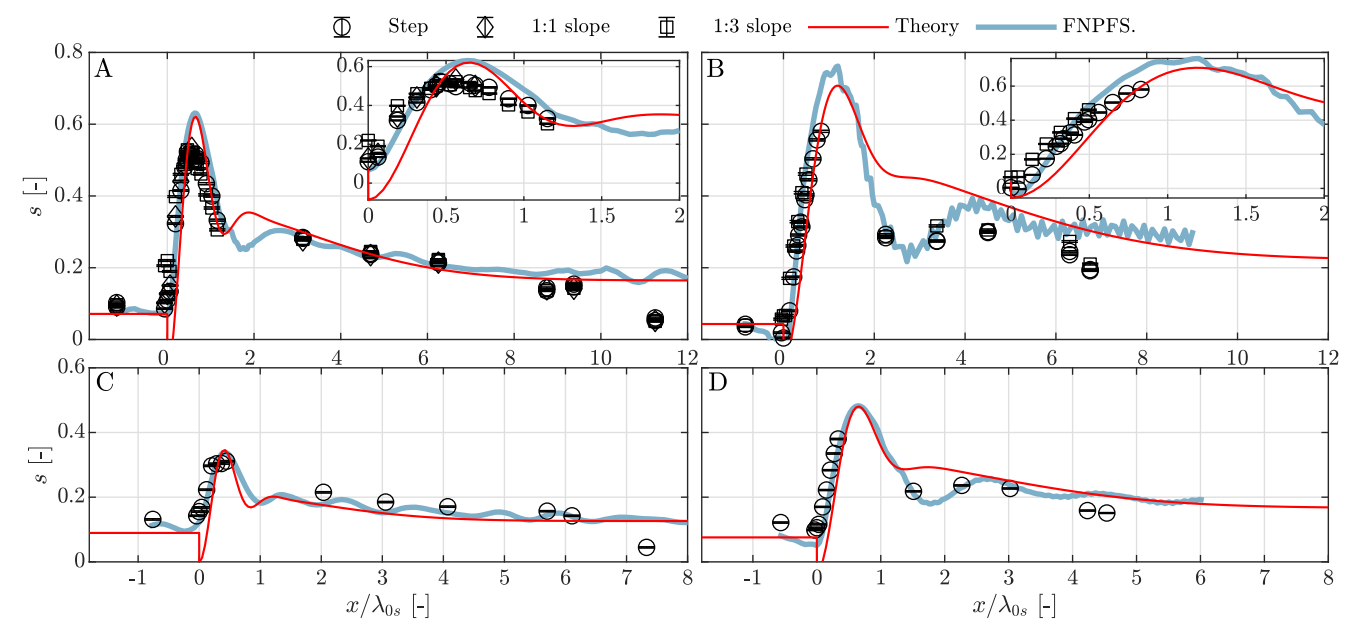

FIGURE 2. Spatial variation of skewness $s$ comparing theoretical prediction by (2.7), experiments and numerical simulations ('FNPFS') with $\lambda_{0 s}$ the peak wavelength (step at $x=0$ ). The (small) error bars for the experiments and the thickness of the blue line for the numerics denote \pm one standard deviation either side of the mean. Panels A-D correspond to the cases in table 1.

parameters presented in Tab. 1. Cases A and C start in deeper water $\left(k_{0} h_{d}=1.6\right)$ compared to $\mathrm{B}$ and $\mathrm{D}\left(k_{0} h_{d}=1.0,1.1\right)$, and cases $\mathrm{A}$ and $\mathrm{B}\left(k_{0} h_{d} /\left(k_{0 s} h_{s}\right)=1.9,1.8\right)$ experience a larger depth transition than $\mathrm{C}$ and $\mathrm{D}\left(k_{0} h_{d} /\left(k_{0 s} h_{s}\right)=1.6,1.5\right)$. We examine skewness and kurtosis (§3.2), followed by wave crest distribution ( $(3.3)$.

For each case in Tab. 1, several realisations with different randomised amplitudes and phases were generated in both experiments and numerical simulations. Random amplitudes and phases are generated for each frequency component as described in $\S 2.2$. Each of these realisations was $\sim 20 \min (1200 \mathrm{~s})$, with frequency spacing $\Delta f=$ $1 / 1800 \mathrm{~Hz}$. The parameters used as input to the statistical model are estimated from the experimental values measured at the first gauge, before the step. For our (narrowbanded) model predictions, we have used an estimated, equivalent Gaussian envelope $A$ to compute the envelope functions $R_{20}(x)$ and $R_{22}(x)$. 


\subsection{Skewness and kurtosis}

Fig. 2 shows the spatial variation of skewness either side of the ADT $(x=0)$ for the four cases in Tab. 1, including the step and the 1:1 and 1:3 slopes (for cases $A$ and B). In all cases, the transition to a higher equilibrium value of skewness associated with larger bound waves for shallower depth is associated with sharp peaks. These sharp peaks, observed in previous studies, occur within one wavelength of the step. Their magnitudes and locations in both experiments and numerics, which are in good agreement, are predicted well by our statistical model. The non-uniform spatial resolution of experimental values is due to the limited number of gauges. We do not observe a significant difference between the experiments for a step and the 1:1 and 1:3 slopes, implying that the physics of steep slopes is captured well by our model. The largest peak is observed when the depth before the step is shallowest and the depth transition is greatest (case B).

The only significant difference between the numerical simulations and our model in Fig. 2 arises in the region after the first peak, where we have only few experimental observations (cases B and D), which agree better with the numerical simulations. These differences are likely due to violation of the narrow bandwidth assumption in our model for the broad-banded JONSWAP spectra we have used in the interest of realism, which causes the smearing out of the super-harmonic beating pattern.

Fig. 3 shows the spatial variation of kurtosis either side of the ADT $(x=0)$ for cases AD. In all cases, the transmission over the ADT is associated with sharp peaks in kurtosis. In principle, two processes can contribute to kurtosis: third-order processes associated with modulational (in)stability that drive build-up of phase correlation of the linear signal (Janssen 2003) on the one hand and second-order bound and free waves on the other hand; both are captured by the experiments and the numerical simulations but only the latter by our statistical model. In the cases that are modulationally unstable before the ADT (A and $\mathrm{C}$ with $k_{0} h_{d}=1.6>1.36$ ), the kurtosis in experiments and numerics starts slightly above the linear Gaussian value of 3 with a negligible contribution by secondorder bound waves. In all cases, the kurtosis in experiments and numerics gradually decays to an equilibrium value slightly below 3 to the right of the ADT corresponding to the modulationally stable conditions on the shallower side $\left(k_{0 s} h_{s}<1.36\right)$, where our second-order accurate statistical model only predicts a small positive contribution to kurtosis by bound waves $\left(R_{20}=R_{22}=0\right.$ for $x \gg \sigma_{0 s}$ with $\left.\sigma_{0 s}=\sigma_{0} c_{g 0 s} / c_{g 0}\right)$.

Crucially, the locations of the peaks in kurtosis are predicted accurately by our (secondorder accurate) model in all cases. So are their magnitudes with the relatively small differences between numerics and our model potentially arising because of third-order effects not included in our model, except for the deepest of our cases (case C, with $k_{s} h_{d}=1.6, k_{0 s} h_{s}=1.0$ ), for which these third-order effects are of the same order of magnitude as the (small) second-order peaks predicted by our model. Peaks are more significant when the water depth is shallower and the depth transition is greater. The kurtosis for the step and the 1:1 and 1:3 slopes is not significantly different.

\subsection{Wave crest distribution}

Fig. 4 shows the exceedence probability distribution and the spatial variation of the probability of rogue waves, defined here as $P\left(\tilde{\zeta}_{c}>1.25 H_{m 0}\right)$ with $H_{m 0}$ the measured significant wave height (of the entire signal) for case A (similar results are obtained for cases B-D). Very good agreement between the experiments, numerical simulations, and theory is evident. In particular, the agreement is clear at the location of maximum rogue wave probability (gauge 5b). Minor differences in the rogue wave probability between, 

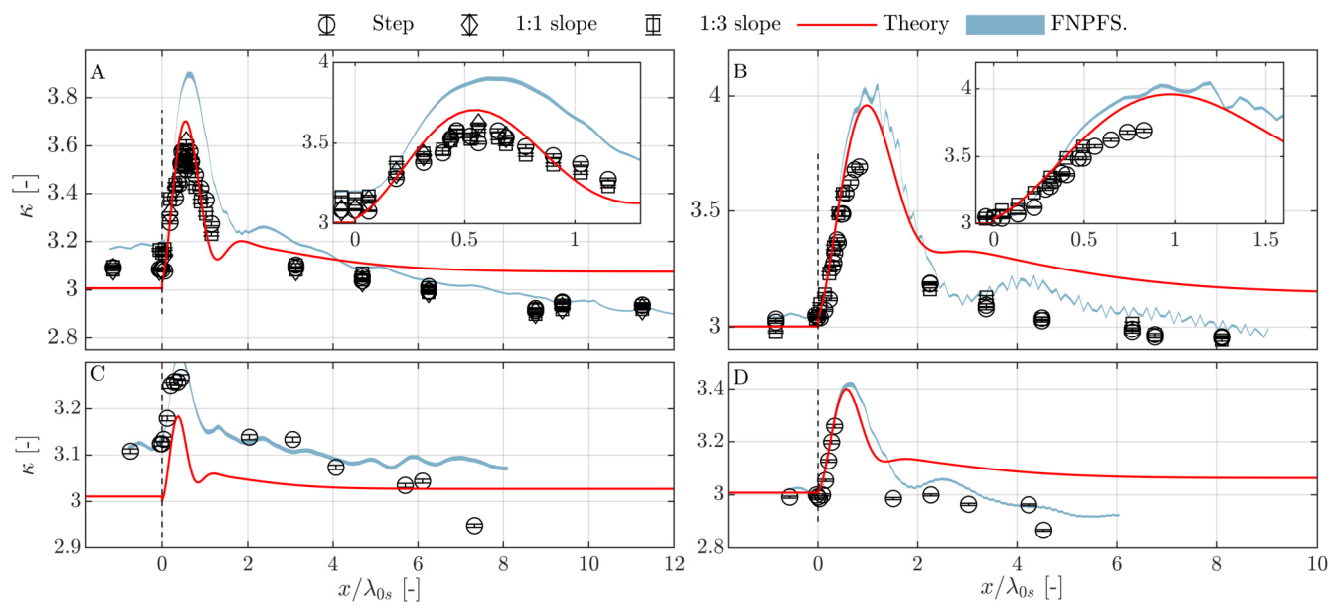

FIGURE 3. Spatial variation of kurtosis $\kappa$ comparing theoretical prediction by (2.8), experiments and numerical simulations ('FNPFS') with $\lambda_{0 s}$ the peak wavelength (step at $x=0$ ). The (small) error bars for the experiments and the thickness of the blue line for the numerics denote \pm one standard deviation either side of the mean. Panels A-D correspond to the cases in table 1.

on the one hand, the theoretical predictions and, on the other hand, the experiments and numerics, which are in better agreement, can be observed close to the ADT $(0<$ $\left.x / \lambda_{0 s} \lesssim 0.2\right)$ and after the peak $\left(1 \lesssim x / \lambda_{0 s} \lesssim 3\right)$. The former is likely due to evanescent waves being neglected in our model, whereas the latter is primarily a result of the narrowbandwidth assumption we have made (see Li et al. (2021b)).

\section{Conclusions}

We have presented a statistical model to explain why rogue waves occur atop abrupt depth transitions (ADTs) in intermediate water depth. The model, based on Massel (1983) and Li et al. (2021b), includes nonlinearity up to second order in steepness, assumes narrow-banded irregular waves, represents the ADTs as a infinitely steep step, and ignores the role of evanescent waves. We have validated our model through comparison to laboratory experiments and a fully nonlinear numerical simulations of the water wave equations. In doing so, we have explained the mechanism behind the sharp peaks in kurtosis, indicative of rogue waves, atop ADTs recently observed by a large number of authors in experiments and numerical simulations (see e.g., Trulsen et al. (2020) and references therein). We show that peaks in kurtosis arise from the co-existence of linear free and second-order bound waves, which are also present in the absence of the $\mathrm{ADT}$, and the second-order free waves additionally generated due to the ADT. As the second-order free waves always overlap with the linear waves near the top of the ADT but travel at different phase and group speeds (from the linear waves), the peaks are localized and the total wave field becomes in-homogeneous. Compared to (realistically broad-banded) numerical simulations and experiments, our (narrow-banded) model provides an accurate prediction of the spatially varying probability distribution of rogue waves and the associated peaks in skewness and kurtosis atop ADTs and identifies and explains a new physical mechanism by which rogue waves can arise in the ocean.

Acknowledgements: We acknowledge support from NSFC-EPSRC-NERC grants 51479114, EP/R007632/1, EP/R007519/1 and a Flexible Fund grant from the UK \& 

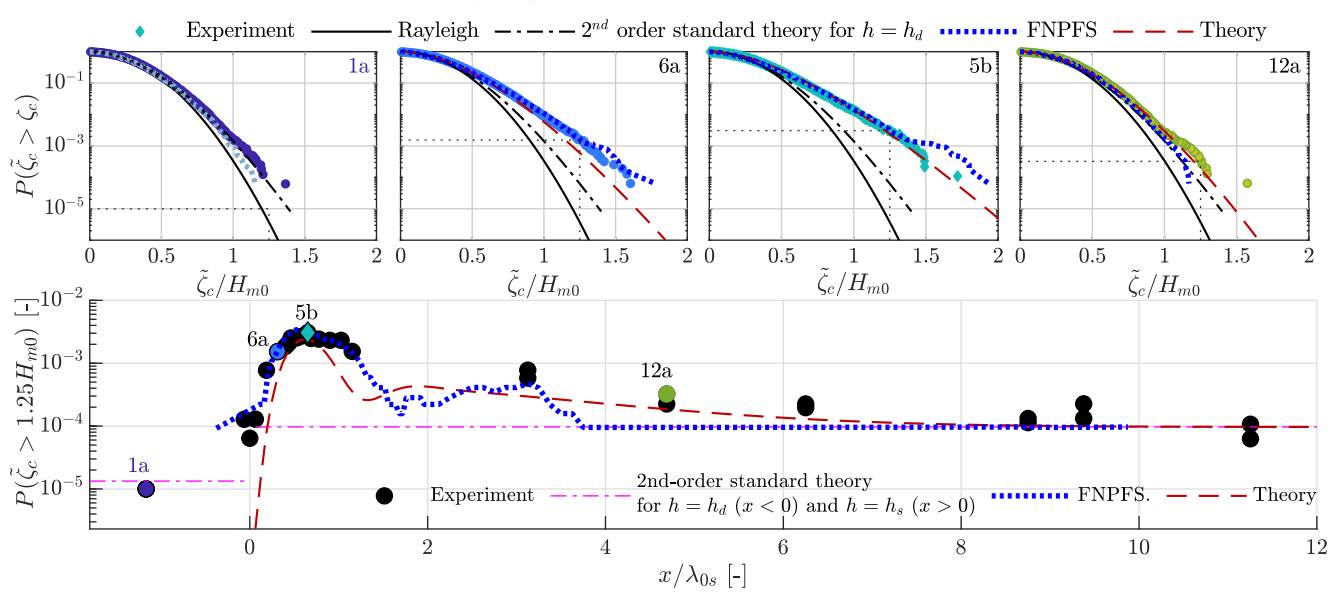

FiguRE 4. Crest exceedance probability distribution at four locations (top) and the probability of rogue waves as a function of space (bottom) for case A, comparing the theoretical prediction by (2.12), experiments and numerical simulations ('FNPFS') (step at $x=0$ ).

China CORE. YL acknowledges the support from the research council of Norway through the FRIPRO mobility project 287389. SD acknowledges a Dame Kathleen Ollerenshaw Fellowship. TSvdB acknowledges a RAEng Research Fellowship. The authors would like to thank staff at the COAST laboratory.

Declaration of interest: The authors report no conflicts of interest.

\section{REFERENCES}

Adcock, T. A. A. \& TAYlor, P. H. 2014 The physics of anomalous ('rogue') ocean waves. Rep. Prog. Phys. 77 (10), 105901.

Benjamin, T. B. \& Feir, J. E. 1967 The disintegration of wave trains on deep water Part 1. Theory. J. Fluid Mech. 27 (3), 417-430.

Bolles, C. T., Speer, K \& Moore, M. N. J. 2019 Anomalous wave statistics induced by abrupt depth change. Phys. Rev. Fluids 4 (1), 011801.

Ducrozet, G. \& Gouin, M. 2017 Influence of varying bathymetry in rogue wave occurrence within unidirectional and directional sea-states. J. Ocean. Eng. Sci. 3 (4), 309-324.

Dudley, John M, Genty, Gö̈ry, Mussot, Arnaud, Chabchoub, Amin \& Dias, Frédéric 2019 Rogue waves and analogies in optics and oceanography. Nat. Rev. Phys. 1 (11), 675689.

Dysthe, K. B., Krogstad, H. E. \& Müller, P. 2008 Oceanic rogue waves. Annu. Rev. Fluid Mech. 40, 287-310.

Fedele, F., Brennan, J., De León, S. P., Dudley, J. \& Dias, F. 2016 Real world ocean rogue waves explained without the modulational instability. Scientific reports $\mathbf{6}, 27715$.

Forristall, George Z 2000 Wave crest distributions: Observations and second-order theory. J. Phys. Oceanogr. 30 (8), 1931-1943.

Gramstad, O., Zeng, H., Trulsen, K. \& Pedersen, G. K. 2013 Freak waves in weakly nonlinear unidirectional wave trains over a sloping bottom in shallow water. Phys. Fluids 25 (12), 122103.

Janssen, P. A. E. M. 2003 Nonlinear four-wave interactions and freak waves. J. Phys. Oceanogr. 33, 863-884.

Kashima, H., Hirayama, K. \& Mori, N. 2014 Estimation of freak wave occurrence from deep to shallow water regions. Coastal Engineering Proceedings 1 (34), 36.

Kharif, C., Pelinovsky, E. \& Slunyaev, A. 2008 Rogue waves in the ocean. Springer Science \& Business Media.

Lawrence, C., Trulsen, K. \& Gramstad, O. 2021 Statistical properties of wave kinematics 
in long-crested irregular waves propagating over non-uniform bathymetry. Phys. Fluids 33 (4), 046601.

Li, Y., Draycott, S., Adcock, T. A. A. \& van den Bremer, T. S. 2021a Surface wavepackets subject to an abrupt depth change. Part II: experimental analysis. J. Fluid Mech. 915 (A72).

Li, Y., Zheng, Y. K.and Lin, Z. L., Adcock, T. A. A. \& van den Bremer, T. S. $2021 b$ Surface wavepackets subject to an abrupt depth change. Part I: second-order theory. $J$. Fluid Mech. 915 (A71).

MA, Y. X., Dong, G. \& MA, X. 2014 Experimental study of statistics of random waves propagating over a bar. Coast. Eng. Proc. 1 (34), 30.

MajdA, A. J., Moore, M. N. J. \& QI, D. 2019 Statistical dynamical model to predict extreme events and anomalous features in shallow water waves with abrupt depth change. Proc. Natl. Acad. Sci. U.S.A 116 (10), 3982-3987.

Massel, S. R. 1983 Harmonic generation by waves propagating over a submerged step. Coast. Eng. 7 (4), 357-380.

Mei, C. C., Stiassnie, M. \& Yue, D. K. P. 1989 Theory and Applications of Ocean Surface Waves: Part 1: Linear Aspects Part 2: Nonlinear Aspects. World Scientific.

Mori, N. \& JAnssen, P. A. E. M. 2006 On kurtosis and occurrence probability of freak waves. J. Phys. Ocean. 36 (7), 1471-1483.

Onorato, M., Residori, S., Bortolozzo, U., Montina, A. \& Arecchi, F. T. 2013 Rogue waves and their generating mechanisms in different physical contexts. Physics Reports 528, $47-89$.

Sergeeva, A., Pelinovsky, E. \& Talipova, T. 2011 Nonlinear random wave field in shallow water: variable Korteweg-de Vries framework. Nat. Hazards Earth Syst. Sci. 11 (2), 323330.

TAyfun, M. A. 1980 Narrow-band nonlinear sea waves. J. Geophys. Res. C: Oceans 85 (C3), $1548-1552$.

TAYfun, M. A. 1986 On narrow-band representation of ocean waves: 1. Theory. J. Geophys. Res. C: Oceans 91 (C6), 7743-7752.

Toffoli, A, Monbaliu, J, Onorato, M, Osborne, AR, Babanin, AV \& BitnerGregersen, E 2007 Second-order theory and setup in surface gravity waves: a comparison with experimental data. J. Phys. Oceanogr. 37 (11), 2726-2739.

Trulsen, K. 2018 Rogue waves in the ocean, the role of modulational instability, and abrupt changes of environmental conditions that can provoke non equilibrium wave dynamics. In The Ocean in Motion, pp. 239-247. Springer.

Trulsen, K., Raustøl, A., Jorde, S. \& Bæverfjord Rye, L. 2020 Extreme wave statistics of long-crested irregular waves over a shoal. J. Fluid Mech. 882.

Trulsen, K., Zeng, H. M. \& Gramstad, O. 2012 Laboratory evidence of freak waves provoked by non-uniform bathymetry. Phys. Fluids 24 (9), 097101.

Viotti, C. \& Dias, F. 2014 Extreme waves induced by strong depth transitions: Fully nonlinear results. Phys. Fluids 26 (5), 051705.

Zeng, H. \& Trulsen, K. 2012 Evolution of skewness and kurtosis of weakly nonlinear unidirectional waves over a sloping bottom. Nat. Hazards Earth Syst. Sci. 12 (3), 631-638.

ZhANG, J. \& BenOIT, M. 2021 Wave-bottom interaction and extreme wave statistics due to shoaling and de-shoaling of irregular long-crested wave trains over steep seabed changes. J. Fluid Mech. 912.

Zhang, J., Benoit, M., Kimmoun, O., Сhabchoub, A. \& Hsu, H. C. 2019 Statistics of extreme waves in coastal waters: Large scale experiments and advanced numerical simulations. Fluids 4 (99), 1-24.

Zheng, Y. K., Lin, Z. L., Li, Y., Adcock, T. A. A., Li, Y. \& van den Bremer, T. S. 2020 Fully nonlinear simulations of extreme waves provoked by strong depth transitions: the effect of slope. Phys. Rev. Fluids 5 (064804). 